\title{
Anti-proliferative and proapoptotic effects of benzyl isothiocyanate on human pancreatic cancer cells is linked to death receptor activation and RasGAP/Rac1 down-modulation
}

\author{
ARUNA BASU and SUBRATA HALDAR \\ Center for Biomedical Sciences, Department of Pharmacology, Case Comprehensive Cancer Center, \\ MetroHealth Campus, Case Western Reserve University, Cleveland, OH, USA
}

Received April 13, 2009; Accepted June 4, 2009

DOI: 10.3892/ijo_00000370

\begin{abstract}
Benzyl isothiocyanate can exert anti-tumor effect by arrest of cell cycle progression and induction of apoptosis in human pancreatic cancer cells. Among them, the dissection of the molecular mechanism of induction of apoptosis is important because the knowledge may be exploited for both cancer prevention and treatment. Our studies reported here indicate that BITC-mediated apoptosis involves the disappearance of intact 21-kDa Bid protein, cytochrome c release and predominant procaspase-3 cleavage. Using adenocarcinoma and metastatic pancreatic cancer cells, we investigated whether this dietary isothiocyanate induces apoptosis by converging two major pathways: the death receptor-mediated extrinsic and the mitochondrial intrinsic pathway. Indeed, cell surface receptor analysis by flow cytometry demonstrates the upregulation of DR4, a member of death receptor family in BITC exposed pancreatic cancer cells. Since BITC is able to trigger death receptor signaling, we were interested in examining the effects of BITC and death receptor ligand TRAIL together on pancreatic cancer cell death. Interestingly, BITC augments TRAIL-induced apoptosis in both metastatic and adenocarcinoma cells. Moreover, we report for the first time that the sensitivity of metastatic pancreatic cancer cells to this isothiocyanate might be due to down-modulation of the proangiogenic molecule small GTPase Rac1 and caspase-3 substrate RasGAP, a regulator of Rho GTPase family.
\end{abstract}

\section{Introduction}

Multiple epidemiological studies as well as experimental animal studies suggest that high intake of cruciferous vegetables might reduce the incidence of cancer (1-10). As a

Correspondence to: Dr Aruna Basu, R455, Rammelkamp Building, MetroHealth Medical Center, 2500 MetroHealth Drive, Cleveland, OH 44109, USA

E-mail: abasu@metrohealth.org

Key words: pancreatic cancer, apoptosis, benzyl isothiocyanate, TRAIL, death receptor, apoptosis result, cruciferous vegetables, a rich source of glucosinolates, have been of great interest for potential use in the chemoprevention of cancer. The glucosinolates are known to be degraded into isothiocyanates by enzymatic action of plantspecific myrosinase or intestinal flora in the body. The biological effects conferred by isothiocyanates (ITC) are selectively targeted towards transformed or malignant cells without affecting normal cells (11). The potential of isothiocyanates to be chemopreventive agents might be attributed to a variety of mechanisms of actions $(2,11)$. Primarily, these compounds are strong inhibitors of the phase I enzymes such as cytochrome $\mathrm{P}_{450}$ activity (12). This cytochrome $\mathrm{P}_{450}$ inhibitory property of isothiocyanates could probably prevent carcinogenicity of $\mathrm{N}$-nitrosobis (2-oxopropyl) amine (BOP) in animals $(13,14)$, because this compound is converted to active form by cytochrome $\mathrm{P}_{450}$ enzymes. Another important biological effect of isothiocyanates has been linked to the increased detoxification of carcinogens by phase II enzymes $(15,16)$.

Among isothiocyanates, the prototypical member benzyl isothiocyanate (BITC) has been shown to perturb cell cycle and apoptosis in pancreatic cancer cells $(3,17-21)$. The multiple mechanisms of the anti-tumor effect of BITC possibly include the modulation of angiogenesis along with the induction of cell death $(22,23)$. Previously, we demonstrated that this chemopreventive agent can invoke cell death by phosphorylation of anti-apoptotic protein $\mathrm{Bcl}-\mathrm{xL}$ as well as activation of both initiator and executioner caspases in a variety of cancer cells (17). Here we report that both death receptors and mitochondrial machinery are involved in BITC-mediated apoptosis of pancreatic cancer cells. Since BITC can up-regulate death receptor DR4, we investigated the effects of BITC and death receptor ligand TRAIL together in cell culture model. TRAIL (tumor necrosis factorrelated apoptosis-inducing ligand) is a type II transmembrane cytokine and an inducer of apoptosis in cancer cells $(24,25)$. Interestingly, BITC can cooperate with TRAIL to enhance the sensitivity of pancreatic cancer cells to apoptosis in vitro. Our report suggests that the sensitivity of pancreatic cancer cells to BITC might be due to down-modulation of the proangiogenic molecule small GTPase Rac1 (26-28) as well as RasGAP, which is a regulator of Rho GTPase family and known to be cleaved by caspase-3 $(29,30)$. 


\section{Materials and methods}

Cell culture and treatment. Human pancreatic cancer cells BxPC-3, CFPAC-1 (adenocarcinoma) and Hs766T (metastatic) were obtained from the American Type Culture Collection (ATCC, VA). BxPC-3 and Hs766T cells were grown in RPMI with supplements of $10 \%$ fetal bovine serum and $50 \mu \mathrm{g} / \mathrm{ml}$ gentamycin at $37^{\circ} \mathrm{C}$ in a $5 \% \mathrm{CO}_{2}$-humidified atmosphere. CFPAC-1 cells were cultured in Iscove's medium with the above supplements. Unless otherwise stated, CFPAC-1, BxPC-3 and Hs766T cells were treated with 2.5, 5 and $7.5 \mu \mathrm{M}$ BITC (LKT Laboratories, MN), respectively, for $24 \mathrm{~h}$.

Western immunoblotting. Following the designated treatment, total cellular proteins were extracted in lysis buffer

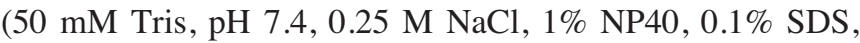
$0.5 \%$ deoxycholate, $1 \mathrm{mM}$ EDTA) containing a protease inhibitor cocktail (Roche Diagnostics, NJ) by repeating freeze-thaw cycle. The resulting lysate was subjected to a total protein assay using the BCA reagent (Pierce Biotechnology, IL). Equal protein from each sample was fractionated by SDS-PAGE and blotted into a nitrocellulose membrane (GE Healthcare, NJ). Membranes were probed with DR-4 (Santa Cruz Biotechnology, CA), Bid (Cell Signaling, MA) PARP, procaspase-3, cytochrome c (BD Biosciences, CA) primary antibodies followed by incubation with HRP-conjugated secondary antibodies. Finally, immunodetection was carried out $(31,32)$ by the enhanced chemiluminescence method (GE Healthcare, NJ). Immunodetection with $B$-Actin antibody (Sigma, MO) served as a protein loading control.

Isolation of mitochondria. Highly enriched mitochondrial fraction was prepared (33) from control and BITC-treated cells using a kit from BioVision (Mountain View, CA). Briefly, cells were washed with PBS and homogenized in extraction buffer (containing dithiothreitol and protease inhibitor) with ice-cold dounce homogenizer. The efficiency of homogenization was microscopically checked. Homogenate was centrifuged at $700 \mathrm{x}$ g for $10 \mathrm{~min}$ at $4^{\circ} \mathrm{C}$. The supernatant was collected and further centrifuged at $10,000 \mathrm{x}$ g for $30 \mathrm{~min}$ at $4{ }^{\circ} \mathrm{C}$. The pellet was collected and suspended in mitochondrial extraction buffer.

Cell surface death receptor analysis. Cells were detached by a non-enzymatic cell dissociation buffer (Sigma-Aldrich) followed by a wash with PBS and re-suspension in ice-cold staining buffer (eBioscience, CA). Next, approximately $0.5 \times 10^{6}$ cells were incubated on ice for $15 \mathrm{~min}$ with $50 \mu \mathrm{g} / \mathrm{ml}$ $\gamma$-globulin (MP Biochemicals, $\mathrm{OH}$ ) and then were stained (25) in the dark for $1 \mathrm{~h}$ with phycoerythrin-conjugated antiFas, anti-DR4, anti-DR5 or immunoglobulin G1 isotype control antibodies (eBioscience). Subsequent to staining, cells were washed three times in staining buffer and analyzed by flow cytometry. A total of 10,000 events were monitored in Epics-XL (Beckman Coulter, CA) at the institute's core facility.

Clonogenic cell survival assay. BxPC-3 cells were treated with BITC alone, TRAIL (R\&D Systems, MN) alone or combination of BITC and TRAIL for $24 \mathrm{~h}$. Control and treated cells were washed two times with phosphate-buffered saline and then seeded in triplicate $(20,000$ cells $/ 10-\mathrm{cm}$ dish). The cells were allowed to grow for an additional 3 weeks in the absence of BITC. Medium was changed every four days. The cells were then fixed with $4 \%$ buffered formalin (Electron Microscopy Sciences, PA) and stained with $0.05 \%$ crystal violet for visualization and photography (34). The number of colonies was counted manually.

\section{Results}

BITC activates proapoptotic protein Bid and triggers cytochrome c release from mitochondria. Among the two major apoptotic pathways such as mitochondrial 'intrinsic' and transmembrane 'extrinsic', the latter one comprises of activation of death receptors (DR) such as Fas, TNF receptor 1, DR4 or DR5 (35). Upon activation by interacting with their respective ligands such as FasL, TRAIL $(24,25,35)$, a signal transduction cascade ensues by the recruitment of DRassociated molecules such as FADD. Subsequent activation of initiator caspases such as caspase- 8 can lead to cleavage of the proapoptotic protein Bid. Truncated Bid (t-Bid) can translocate to mitochondria and can orchestrate mitochondrial events that can result in biochemical as well as morphological alterations implicated with programmed cell death. Primarily, we investigated the status of Bid protein in metastatic pancreatic cancer cells Hs766T following BITC exposure. Notably, we observed that BITC treatment as early as $16 \mathrm{~h}$ leads to significant disappearance of intact $21 \mathrm{kDa} B i d$ from total cellular extract of Hs766T and BxPC-3 cells (Fig. 1A). The cleavage of proapoptotic protein Bid provides us an important clue regarding the cross talk between death receptor and mitochondria (35-37) during BITC-mediated demise of pancreatic carcinoma cells. The interaction of truncated Bid with Bax, either in the cytosol or on the mitochondrial surface, renders conformational changes in Bax. Proapoptotic Bax then inserts itself into outer mitochondrial membrane where it oligomerizes and facilitates release of cytochrome c from the intermembrane mitochondrial space. We determined the level of cytochrome $\mathrm{c}$ in highly enriched mitochondrial fraction isolated from control and BITC-treated BxPC-3 cells. Fig. 1B shows the depletion of cytochrome c from mitochondrial fraction following BITC exposure of pancreatic cancer cells.

BITC-mediated cell death is correlated with TRAIL receptor (DR4) up-regulation. We previously reported that BITC can activate caspase- 8 in pancreatic cancer cells (17). Caspase- 8 is known to be down-stream of death receptor signaling (35). Indeed, by cell surface death receptor analysis using flow cytometry, we observed substantial increase in the expression of death receptor, DR4 (Fig. 2B) due to BITC treatment, when compared with the respective control (Fig. 2A). However, little effect was noted in the case of other death receptors such as DR5 or Fas. As shown in Fig. 2C, immunoblot analysis of whole cell extract with DR4 antibody also revealed an increase of DR4 protein following BITC exposure.

Combination treatment of BITC and death receptor ligand TRAIL diminishes survival and enhances apoptosis of 
A.

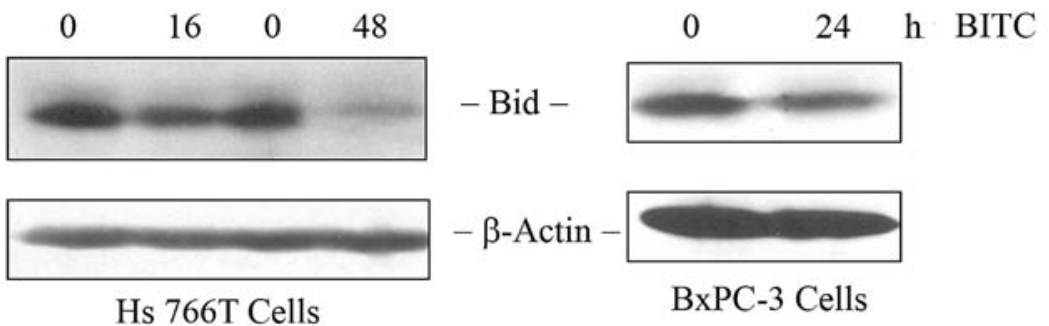

B.

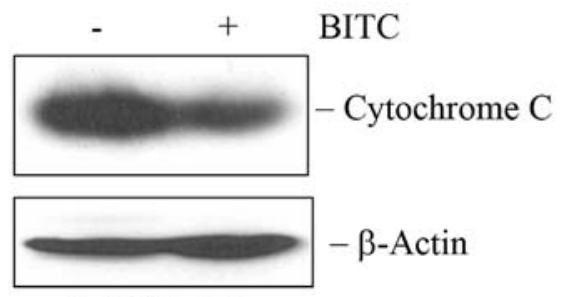

BxPC-3 Cells

Figure 1. BITC-mediated alteration in apoptotic regulators. (A) Decrease in $21 \mathrm{kDa}$ Bid protein in the total cellular extract of BITC-challenged pancreatic cancer cells. Pancreatic cancer cells BxPC-3 and Hs766T were exposed to 5 and $7.5 \mu \mathrm{M}$ BITC, respectively, for the designated period. Total cellular extract was subjected to immunoblot analysis with Bid peptide polyclonal antibody. (B) Release of cytochrome c from mitochondria due to BITC treatment. Mitochondrial fractions isolated from control and BITC-treated BxPC-3 cells were processed for immunoblot analysis with cytochrome $\mathrm{c}$ antibody.

\section{A. Control}
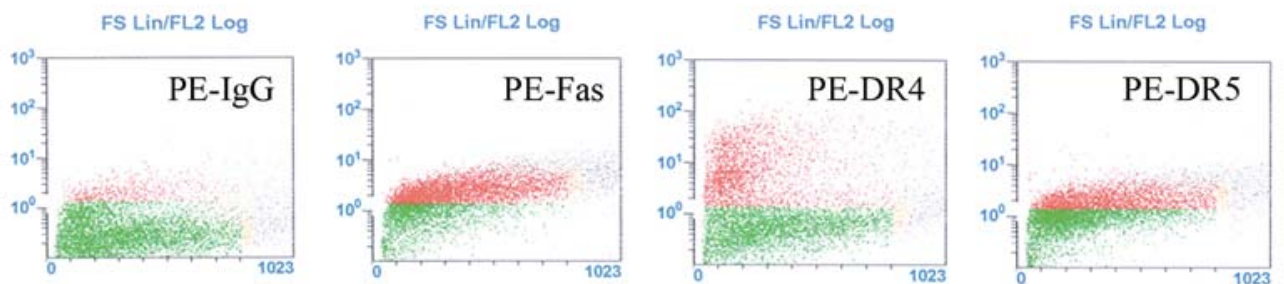

\section{B. BITC Treated}

FS Lin/FL2 Log
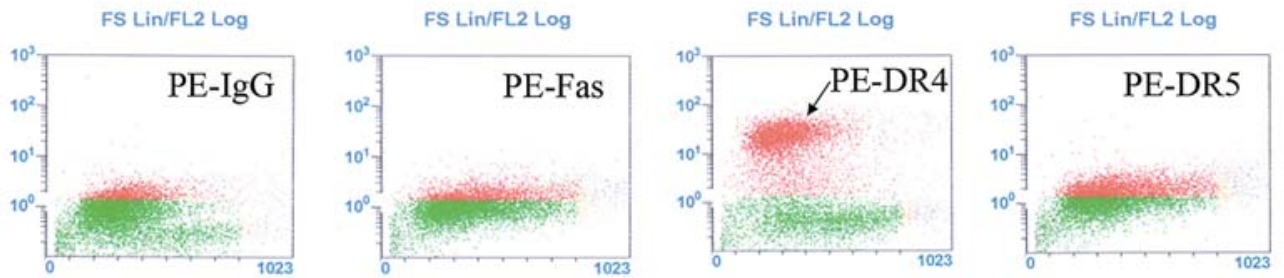

C.

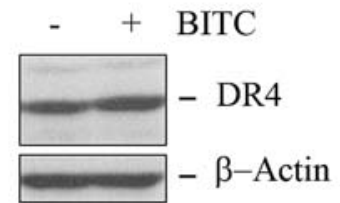

Figure 2. Modulation of death receptors in BITC-exposed pancreatic cancer cells. (A) and (B) Dot plot of expression of cell surface death receptors. Control and $5 \mu \mathrm{M}$ BITC-challenged ( $24 \mathrm{~h}) \mathrm{BxPC}-3$ cells were processed for flow cytometric analysis of surface death receptor using phycoerythrin (PE)-conjugated anti-Fas, anti-DR4, anti-DR5 and IgG1 isotype control antibodies. (C) Western blot analysis of control and BITC-treated pancreatic cancer cell lysate with DR4 antibody.

pancreatic cancer cells. The observation implicating death receptor up-regulation due to BITC treatment provided the rationale to examine the effect of a death receptor ligand such as TRAIL in combination with BITC on human pancreatic cancer cells. The synergistic effect of BITC and TRAIL on pancreatic cancer cell growth was assessed following exposure to BITC or recombinant human TRAIL or BITC and TRAIL together for $16 \mathrm{~h}$. As evident from the clonogenic survival assay (Fig. 3A), the number of colonies in BITC- or TRAILtreated $\mathrm{BxPC}-3$ cells was higher than those formed by 
A. BxPC-3 Cells
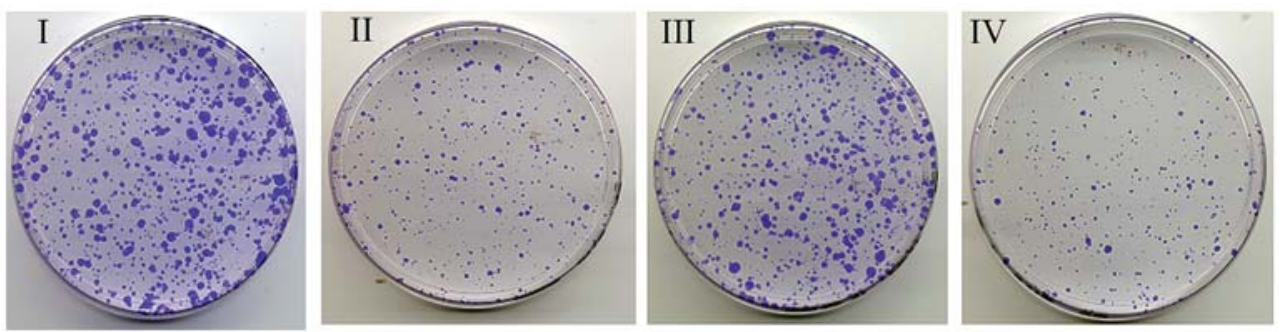

B.

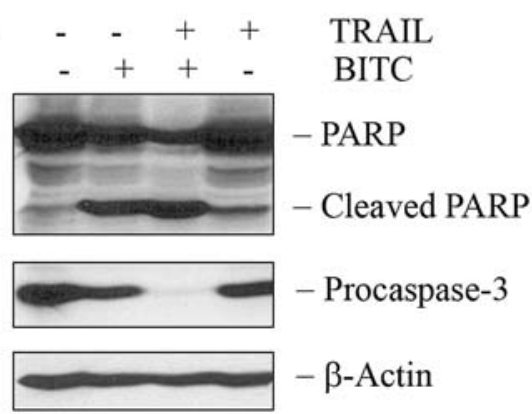

Figure 3. Combinatorial effect of BITC and TRAIL on pancreatic cancer cell survival and apoptosis. (A) Decreased clonogenic survival of pancreatic adenocarcinoma cells due to combinatorial treatment of BITC and TRAIL. Clonogenic cell survival assay was employed to determine the long-term survival ability of BxPC-3 cells in response to several treatment regimens as follows. Panel I, control (DMSO); panel II, BITC ( $2 \mu \mathrm{M})$; panel III, TRAIL (10 ng/ml); panel IV, BITC $(2 \mu \mathrm{M})$ and TRAIL $(10 \mathrm{ng} / \mathrm{ml})$ together. (B) Increased apoptosis due to treatment with BITC and TRAIL together. Following designated treatment, cell lysate was prepared and subjected to Western blot analysis using monoclonal antibody against PARP and procaspase-3.

A.

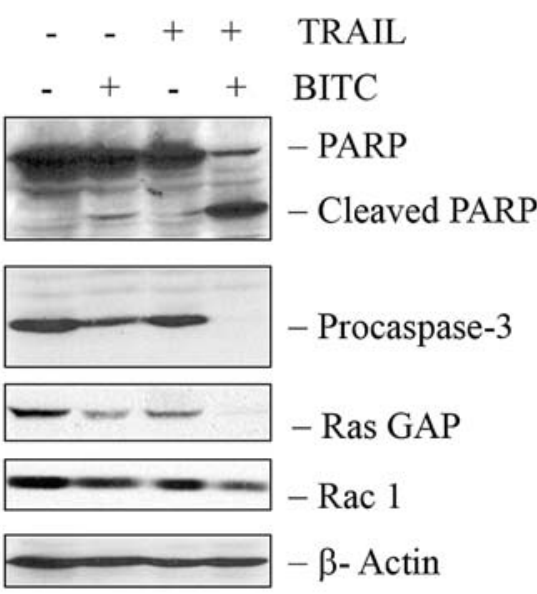

Hs 766T Cells

C.

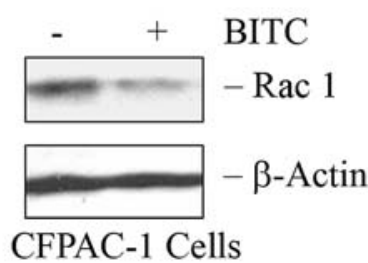

B.

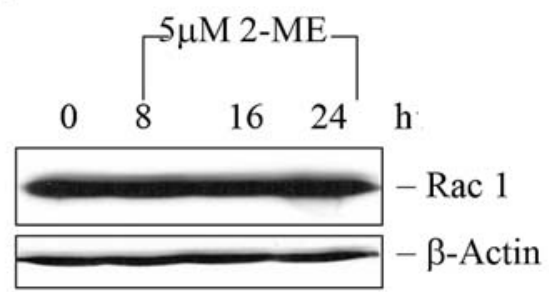

Hs 766T Cells

D.

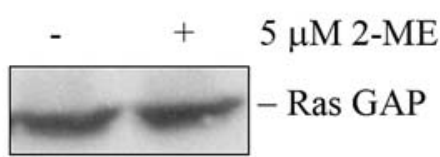

Hs 766T Cells

Figure 4. BITC-mediated apoptosis is associated with down-regulation of RasGAP and Rac1 proteins. (A) Hs766T cells were exposed to either BITC (7.5 $\mu \mathrm{M}$ ) or $10 \mathrm{ng} / \mathrm{ml}$ recombinant human TRAIL or BITC $(7.5 \mu \mathrm{M})$ and $10 \mathrm{ng} / \mathrm{ml}$ TRAIL together for $16 \mathrm{~h}$. Subsequently, cells were processed for immunoblot analysis . (B) Level of Rac1 protein in control and BITC- $(2.5 \mu \mathrm{M})$ treated CFPAC-1 cells. (C) and (D) Immunoblot analysis of control and 2-methoxyestradiol-treated Hs766T cells with Rac1 and RasGAP antibodies. Equal protein loading was verified by Ponceau S staining of the blot. 


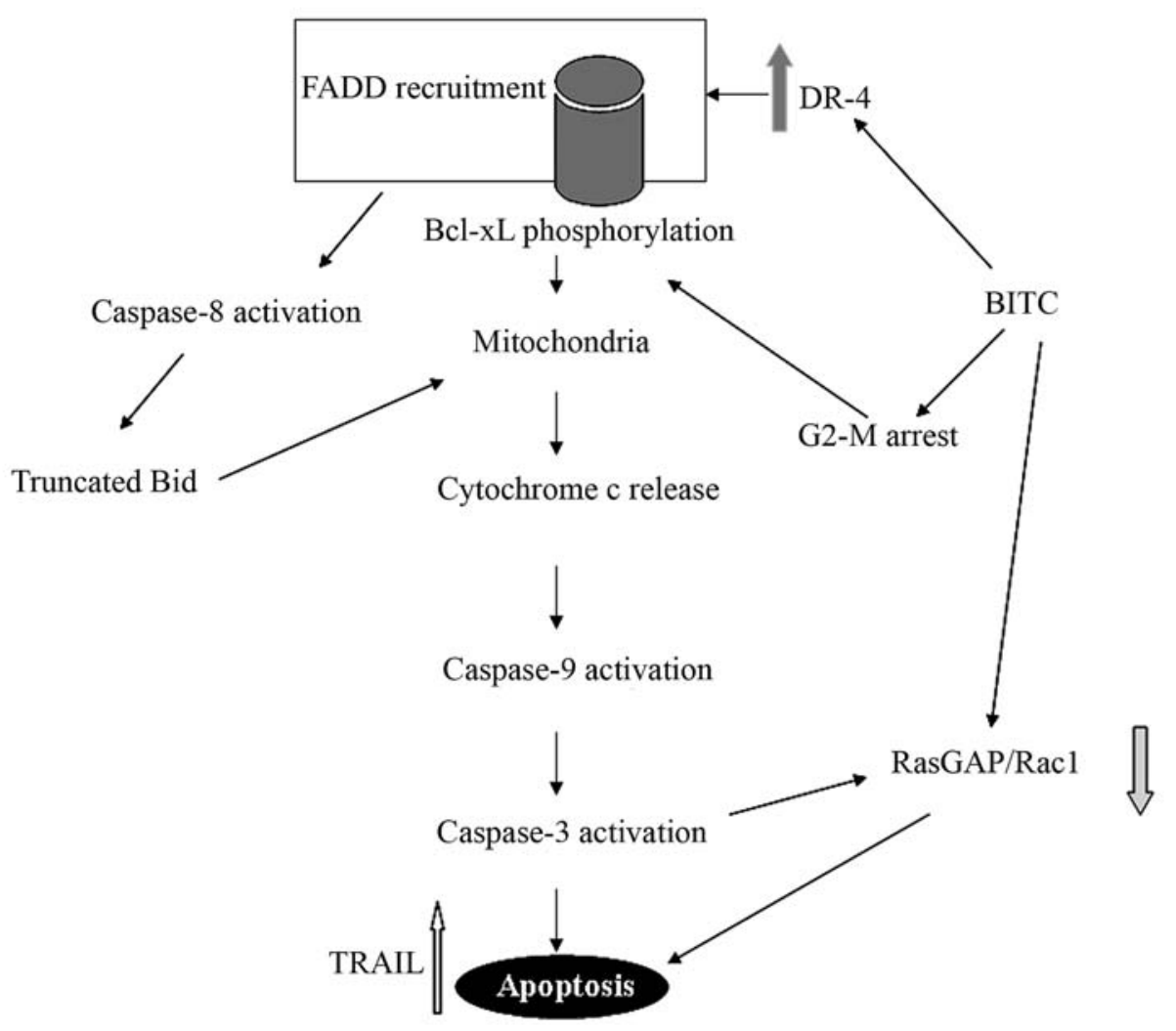

Figure 5. Schematic illustration of the apoptotic signaling pathway induced by BITC in pancreatic cancer cells.

combination treatment (panel IV vs. panels II and III, Fig. 3A). Due to combination treatment, the number of large colonies was reduced approximately $50 \%$ compared to that of BITC treatment alone. Of note, there was no significant variations in the number of abortive colonies (small colonies that did not progress to larger colonies) between the two treatment groups (panel IV vs. panels II, Fig. 3A).

Next, we were interested to examine whether the combination therapy would exert any proapoptotic effect on these cells. In correlation with the decreased cell survival, the combination treatment of BITC and TRAIL was very effective in causing enhanced poly-ADP ribose polymerase (PARP) cleavage, a hallmark of apoptosis, and the activation of caspase-3 in BxPC-3 cells (Fig. 3B). The synergistic action of BITC and TRAIL in mediating increased apoptosis of pancreatic cancer cells thus supports a broad mechanism that involves the up-regulation of death receptor DR4 by BITC.

BITC-mediated proapoptotic effect on metastatic pancreatic cancer cells is correlated with down-regulation of RasGAP and Racl. As shown in Fig. 4A, the potential therapeutic synergy by combining BITC and TRAIL was noted by significant apoptosis of metastatic pancreatic cancer cells Hs766T. Apoptosis was determined by analysis of biochemical markers such as PARP cleavage or caspase- 3 activation. The combination treatment of BITC and TRAIL was very effective for enhanced PARP cleavage as well as for the disappearance of procaspase-3.

Our previous findings $(25,33)$ indicate that metastatic pancreatic cancer Hs766T cells are generally insensitive to anti-cancer agents such as 2-methoxyestradiol (2-ME). However, in 2-ME responsive pancreatic carcinoma MIA$\mathrm{PaCa}-2$ cells, the induction of apoptosis was associated with the attenuation of the level of a member of Rho GTPase family proteins, Rac1 (31). It is known that the elevated expression level of Rho GTPases is linked to malignant transformation and metastasis (26). Since BITC can exert death-inducing effect on metastatic pancreatic cancer cells, we were interested to examine whether this chemopreventive agent can mediate apoptosis by diminishing proangiogenic molecule Rac1. As shown in Fig. 4A, down-regulation of Rac1 is clearly evident when cells are treated with BITC as compared to control. Moreover, we observed significant attenuation of Rac1 protein level in cells treated with BITC and TRAIL together. The attenuation of Rac1 is also evident in BITC-treated pancreatic ductal carcinoma cells CFPAC-1 (Fig. 4B). Previously we showed that under identical condition of BITC treatment, CFPAC-1 cells undergo apoptosis (17).

Another interesting observation emerging from this study is that BITC can down-modulate RasGAP, the main regulator of RasGTPase family members (Fig. 4A). Notably, caspase-3 activation as well as PARP cleavage is correlated with the disappearance of Rac1/RasGAP protein in BITC-treated metastatic pancreatic cancer cells. In contrast, the other chemotherapeutic agent 2-ME which cannot potentiate apoptosis in Hs766T cells, is also unable to alter either Rac1 or RasGAP expression in these cells (Figs. 4C and D). That BITC can attenuate RasGAP with activation of caspases is in conformity with the previous observation demonstrating RasGAP as molecular target of caspases (29). 


\section{Discussion}

Approximately 33,000 new cases of pancreatic cancer occur annually in the United States with approximately the same number of deaths $(38,39)$. Pancreatic ductal adenocarcinoma is the most common pancreatic neoplasm and evolves from a progressive cascade of cellular, morphological and architectural changes from normal ductal epithelium. The late diagnosis, rapid disease progression and resistance to conventional therapy are the major contributing factors for high mortality of pancreatic cancer patients (39). On this basis identifying novel ways of treating and preventing pancreatic cancer is important.

The multiple mechanisms of the anti-tumor effect of BITC possibly include a modulation of angiogenesis along with induction of cell death. BITC might behave as an agent that might intervene simultaneously at several targets in the carcinogenic process.

Previously, we observed that anti-proliferative action of BITC might be due to induction of apoptosis by the activation of both initiator and executioner caspases. Thus it is likely that death receptor signaling (35) and mitochondrial machinery $(36,37)$ converge to execute BITC-induced cell death. Indeed, the cleavage of proapoptotic protein Bid (as demonstrated by the disappearance of intact $21 \mathrm{kDa} \mathrm{Bid}$ ), cytochrome c release from mitochondria, enhancement of cell surface death receptor DR4 and the synergistic anti-proliferative action of BITC and death receptor ligand TRAIL, suggest crosstalk between the death receptor and mitochondria during BITCmediated apoptosis of pancreatic cancer cells. Notably, we report here for the first time that DR4 could be a novel biomarker for BITC-triggered apoptosis. The molecular mechanism of up-regulation of members of the death receptor family following BITC treatment might be attributed to AP1-mediated transcriptional regulation of death receptor. This stems from our previous study implicating a link between JNK pathway and BITC-mediated cell death (17). It is known that activated JNK might lead to increase in AP-1 expression $(40,41)$.

Another important component of our observation is the attenuation of Rho GTPase family protein Rac1 following BITC treatment. Activation of membrane localized Rac1 leads to its binding to effector molecules and subsequent activation of cellular responses such as cell motility, cell adhesion and cell cycle progression. These cellular responses can contribute to malignant transformation, and it has been shown that metastatic phenotype might arise from the mutated form of Rac1. It is noteworthy that in pancreatic adenocarcinoma aberrant expression of Rac1 was observed previously (27). Moreover Rac1-depedent secretion and activation of MMP-2 has been reported to be associated with pancreatic cancer cell invasion (42). Our finding that BITC can downmodulate Rac1 to evoke cell death has potential from therapeutic standpoint. In line with this observation Senger et al (28) also reported that apoptosis of human glioma cells can be induced by suppression of Rac1 activity.

To the best of our knowledge, the potential therapeutic benefit of synergistic treatment of BITC and TRAIL was never explored before for cancer cells. Our results described here indicate that combination treatment regimen (BITC and
TRAIL together) can bolster the apoptotic death of TRAILresistant metastatic pancreatic cancer cells. As depicted in Fig. 5, we report here for the first time the hierarchy of molecular events involving death receptor DR4 and novel modulators Rac1/RasGAP with BITC-mediated demise of pancreatic cancer cells.

\section{Acknowledgements}

The work was partially supported by NIH Grants CA137476 (A. Basu) and CA 109181 (S. Haldar). We would like to thank Flow Cytometry Core Facility of the Comprehensive Cancer Center of Case Western Reserve University Hospitals of Cleveland (NIH Grant CA43703). We also thank D. Haldar and $\mathrm{K}$. Haas for experimental assistance.

\section{References}

1. Hecht SS: Chemoprevention of cancer by isothiocyanates, modifiers of carcinogen metabolism. J Nutr 129: 768S-774S, 1999.

2. Hecht SS: Inhibition of carcinogenesis by isothiocyanates. Drug Metab Rev 32: 395-411, 2000

3. Keum YS, Jeong WS and Kong AN: Chemopreventive functions of isothiocyanates. Drug News Perspect 18: 445-451, 2005.

4. Shukla S and Gupta S: Dietary agents in the chemoprevention of prostate cancer. Nutr Cancer 53: 18-32, 2005.

5. Wattenberg LW: Inhibitory effects of benzyl isothiocyanate administered shortly before diethylnitrosamine or benzo(a)pyrene on pulmonary and forestomach neoplasia in $\mathrm{A} / \mathrm{J}$ mice. Carcinogenesis 8: 1971-1973, 1987.

6. Hecht SS, Isaacs S and Trushin N: Lung tumor induction in A/J mice by the tobacco smoke carcinogens 4-(methylnitrosamino)1-(3-pyridyl)-1-butanone and benzo(a)pyrene: a potentially useful model for evaluation of chemopreventive agents . Carcinogenesis 15: 2721-2725, 1994.

7. Nakamura Y and Miyoshi N: Cell death induction by isothiocyanates and their underlying molecular mechanisms. Biofactors 26: 123-134, 2006.

8. Steck SE, Gammon MD, Hebert JR, Wall DE and Zeisel SH: GSTM1, GSTT1, GSTP1, and GSTA1 polymorphisms and urinary isothiocyanate metabolites following broccoli consumption in humans. J Nutr 7: 904-909, 2007.

9. Kirsh VA, Peters U, Mayne ST, Subar AF, Chatterjee N, Johnson CC and Hayes RB: Prostate, lung, colorectal and ovarian cancer screening trial: prospective study of fruit and vegetable intake and risk of prostate cancer. J Natl Cancer Inst 99: 1200-1209, 2007

10. Chen YR, Wang W, Kong AN and Tan TH: Molecular mechanisms of c-Jun N-terminal kinase-mediated apoptosis induced by anticarcinogenic isothiocyanates. J Biol Chem 273: 1769-1775, 1998 .

11. Zhang Y, Yao S and Li J: Vegetable-derived isothiocyanates: anti-proliferative activity and mechanism of action. Proc Nutr Soc 65: 68-75, 2006.

12. Von Weymarn LB, Chun JA and Hollenberg PF: Effects of benzyl and phenethyl isothiocyanate on P450s 2A6 and 2A13: potential for chemoprevention in smokers. Carcinogenesis 27 : 782-790, 2005.

13. Hecht SS, Trushin N, Rigotty J, Carmella SG, Borukhova A, Akerkar S and Rivenson A: Complete inhibition of 4(methylnitrosamino)-1-(3-pyridyl)-1-butanone-induced rat lung tumorigenesis and favorable modification of biomarkers by phenyl isothiocyanate. Cancer Epidemiol Biomarkers Prev 5: 645-652, 1996.

14. Smith TJ, Guo Z, Li C, Ning SM, Thomas PE and Yang CS: Mechanisms of inhibition of 4-(methylnitrosoamino)-1-(3pyridyl)-1-butanone bioactivation in mouse by dietary phenethyl isothiocyanate. Cancer Res 53: 3276-3282, 1993.

15. Gao X, Dinkova-Kostova AT and Talalay P: Powerful and prolonged protection of human retinal pigment epithelial cells, keratinocytes, and mouse leukemia cells against oxidative damage: the indirect antioxidant effects of sulforaphane. Proc Natl Acad Sci USA 98: 15221-15226, 2001. 
16. Rose P, Faulkner K, Williamson G and Mithen RF: 7 Methylsulfinylheptyl and 8-methylsulfinyloctyl isothiocyanates from watercress are potent inducers of phase II enzymes. Carcinogenesis 21: 1983-1988, 2000.

17. Basu A and Haldar S: Dietary isothiocyanate mediated apoptosis of human cancer cells is associated with Bcl-xL phosphorylation. Int J Oncol 33: 657-663, 2008.

18. Huang C, Ma WY, Li J, Hecht SS and Dong Z: Essential role of p53 in phenethyl isothiocyanate-induced apoptosis. Cancer Res 58: 4102-4106, 1998.

19. Sahu RP and Srivastava SK: The role of STAT-3 in the induction of apoptosis in pancreatic cancer cells by benzyl isothiocyanate. J Natl Cancer Inst 101: 176-193, 2009.

20. Xiao D and Singh SV: Phenethyl isothiocyanate-induced apoptosis in p53-deficient PC-3 human prostate cancer cell line is mediated by extracellular signal-regulated kinases. Cancer Res 62: 3615-3619, 2002.

21. Miyoshi N, Uchida K, Osawa T and Nakamura Y: A link between benzyl isothiocyanate-induced cell cycle arrest and apoptosis: involvement of mitogen-activated protein kinases in the Bcl-2 phosphorylation. Cancer Res 64: 2134-2142, 2004

22. Juge N, Mithen RF and Traka M: Molecular basis for chemoprevention by sulforaphane: a comprehensive review. Cell Mol Life Sci 64: 1105-1127, 2007.

23. Hwang ES and Lee HJ: Benzyl isothiocyanate inhibits metalloproteinase-2/9 expression by suppressing the mitogenactivated protein kinase in SK-Hep1 human hepatoma cells. Food Chem Toxicol 46: 2358-2364, 2008

24. Guo F, Sigua C, Tao J, Bali P, George P, Li Y, Wittmann S, Moscinski L, Atadja P and Bhalla K: Cotreatment with histone deacetylase inhibitor LAQ824 enhances Apo2L/tumor necrosis factor-related apoptosis inducing ligand-induced death inducing signaling complex activity and apoptosis of human acute leukemia cells. Cancer Res 64: 2580-2589, 2004.

25. Basu A, Castle VP, Bouziane M, Bhalla K and Haldar S: Crosstalk between extrinsic and intrinsic cell death pathways in pancreatic cancer: synergistic action of estrogen metabolite and ligands of death receptor family. Cancer Res 66: 4309-4318, 2006.

26. Del Peso L, Hernández-Alcoceba R, Embade N, Carnero A, Esteve P, Paje C and Lacal JC: Rho proteins induce metastatic properties in vivo. Oncogene 15: 3047-3057, 1997.

27. Crnogorac-Jurcevic T, Efthimiou E, Capelli P, Blaveri E, Baron A, Terris B, Jones M, Tyson K, Bassi C, Scarpa A and Lemoine NR: Gene expression profiles of pancreatic cancer and stromal desmoplasia. Oncogene 20: 7437-7446, 2001.
28. Senger DL, Tudan C, Guiot MC, Mazzoni IE, Molenkamp G, LeBlanc R, Antel J, Olivier A, Snipes GJ and Kaplan DR: Suppression of Rac activity induces apoptosis of human glioma cells but not normal human astrocytes. Cancer Res 62: 2131-2140, 2002.

29. Bartling B, Yang J-Y, Michod D, Widmann C, Lewensohn R and Zhivotovsky B: RasGTPase-activating protein is a target of caspases in spontaneous apoptosis of lung carcinoma cells and in response to etoposide. Carcinogenesis 25: 909-921, 2004.

30. Donovan S, Shannon KM and Bollag G: GTPase activating proteins: critical regulators of intracellular signaling. Biochim Biophys Acta 1602: 23-45, 2002.

31. Basu A and Haldar S: 2-Methoxyestradiol mediated signaling network in pancreatic cancer. Front Biosci 14: 2170-2178, 2009

32. Qanungo S, Das M, Haldar S and Basu A: Epigallocatechin-3gallate induces mitochondrial membrane depolarization and caspase-dependent apoptosis in pancreatic cancer cells. Carcinogenesis 26: 958-967, 2005.

33. Qanungo S, Basu A, Das M and Haldar S: 2-Methoxyestradiol induces mitochondria dependent apoptotic signaling in pancreatic cancer cells. Oncogene 21: 4149-4157, 2002.

34. Munshi A, Hobbs M and Meyn RE: Clonogenic cell survival assay. Methods Mol Med 110: 21-28, 2005.

35. Ashkenazi A and Dixit VM: Death receptors: signaling and modulation. Science 281: 1305-1308, 1998.

36. Wang X: The expanding role of mitochondria in apoptosis. Genes Dev 15: 2922-2933, 2001.

37. Green DR and Reed JC: Mitochondria and apoptosis. Science 281: 1309-1312, 1998.

38. Jamal A, Siegel R, Ward E, Murray T, Xu J and Thun MJ: Cancer Statistics. CA Cancer J Clin 57: 43-66, 2007.

39. Goggins M: Identifying molecular markers for the early detection of pancreatic neoplasia. Semin Oncol 34: 303-310, 2007.

40. Li XR, Chong AS, Wu J, Roebuck KA, Kumar A, Parrillo JE Rapp UR, Kimberly RP, Williams JW and Xu X: Transcriptional regulation of Fas gene expression by GA-binding protein and AP-1 in T cell antigen receptor CD3 complex-stimulated T cells. J Biol Chem 274: 35203-35210, 1999.

41. Guan B, Yue P, Lotan R and Sun S-Y: Evidence that the death receptor 4 is regulated by activator protein 1 . Oncogene 21 : 3121-3129, 2002.

42. Binker MG, Binker-Cosen AA, Richards D, Oliver B and Cosen-Binker LI: EGF promotes invasion by PANC-1 cells through Rac1/ROS-dependent secretion and activation of MMP-2. Biochem Biophys Res Commun 379: 445-450, 2009. 